\title{
Lift and Drainage of Electrically Conducting Power Law Fluid on a Vertical Cylinder
}

\author{
K. N. Memon ${ }^{1, *}$, S. Islam ${ }^{2}$, A. M. Siddiqui ${ }^{3}$, Sher Afzal Khan ${ }^{4, *}$, Nazir Ahmad Zafar ${ }^{5}$ and M. Akram ${ }^{1}$ \\ ${ }^{1}$ COMSATS Institute of Information Technology, Islamabad, Pakistan \\ 2 Department of Mathematics, Abdul Wali Khan University Mardan, Pakistan \\ ${ }^{3}$ Pennsylvania State University, York Campus, Edecomb 17403, USA \\ ${ }^{4}$ Department of Computer Science, Abdul Wali Khan University Mardan, Pakistan \\ ${ }^{5}$ College of Computer Science and Information Technolog, King Faisal University, Al Hassa, Saudi Arabia
}

Received: 22 May. 2013, Revised: 5 Sep. 2013, Accepted: 6 Sep. 2013

Published online: 1 Jan. 2014

\begin{abstract}
In this work, the theoretical study of steady flow for lift and drainage of Power law MHD fluid on a vertical cylinder is presented. The governing nonlinear differential equation has been derived from the momentum equation. The resulting equation is then solved using Perturbation method. Series solutions have been obtained for velocity, flow rate and average velocity in both cases. The graphical results for velocity profile is discussed and examined for different parameters of interest. Without MHD our problem reduces to well known Newtonian and Power law problem.
\end{abstract}

Keywords: Thin film flow; Power law MHD fluid; Analytical solution

\section{Introduction}

In recent years, the flow of non-Newtonian fluids has gained considerable attention because of its applications in various branches of science, engineering, and technology: particularly in material processing, chemical industries, and bioengineering. It is an established fact that the flow characteristics of non-Newtonian fluids are quite different when compared with the linearly viscous fluids. Therefore, the well known Navier-Stokes equations are not suitable to explain the behavior of non-Newtonian fluids. Similar to linearly viscous fluids it is difficult to recommend a single model which exhibits all properties of non-Newtonian fluids. Therefore a number of models have been proposed to characterize the non-Newtonian fluid behavior $[1,4]$.

In the category of non-Newtonian fluids the power law model have been extensively studied because of mathematical simplicity and wide spread industrial applications. During the last four decades significant progress has been made in the development of analytical solution and numerical algorithms of power law fluid flow problems $[5,8]$.

Our main focus in this work is on the study of thin film flow for a non-Newtonian fluid with MHD fluid properties. In a thin film flow, the fluid is partially bounded by a solid wall while the other surface is free to interact with another fluid, e.g., air. There are three main conditions which form basis for the formulation of thin films, namely, surface tension, centrifugal forces and gravitational forces. The analysis of thin film flow is important for designing chemical processing equipment. Probably the most striking daily life examples are rain water running down along a window and the flow of a paint down a wall. Study of thin film flows have established significant interest because of its realistic applications in physical and biological sciences $[9,10]$. There are many engineering applications where thin film flow shows the viscoelastic effects and MHD was originally applied to astrophysical and geophysical problems, where it is still very important, but more recently to the problem of fusion power, where the application is the creation and containment of hot plasmas by electromagnetic forces, since material walls would be destroyed. Astrophysical problems include solar structure, especially in the outer layers, the solar wind bathing the earth and other planets, and interstellar magnetic fields. The primary geophysical problem is

\footnotetext{
*Corresponding author e-mail: kamrannazir025@yahoo.com, sher.afzal@awkum.edu.pk
} 
planetary magnetism, produced by currents deep in the planet, a problem that has not been solved to any degree of satisfaction.

Here, in this paper, fluid is considered visco-inelastic with the viscosity function conforming to power law MHD fluid. We examine the thin film flow of a Power law MHD fluid for lift and drainage problems on a vertical cylinder. Two cases are discussed, namely, Newtonian and non-Newtonian respectively. To the best of our knowledge the analytical solution has not been reported elsewhere.

This letter is organized as follows. Section 2 contains the governing equation of power law fluid model. In section 3 the problem under consideration is formulated and solution for the lifting case is given section 4 is reserved for the solution of the drainage case and section 5 results and discussion. In Section 6 concluding remarks are given.

\section{Basic Equations}

The basic equations, governing the flow of incompressible power law MHD fluid neglecting the thermal effects, are:

$$
\begin{aligned}
\nabla . \mathbf{V} & =0 . \\
\rho \frac{D \mathbf{V}}{D t} & =\rho \mathbf{f}-\nabla p+\operatorname{div} \mathbf{S}+(\mathbf{J} \times \mathbf{B}),
\end{aligned}
$$

where $\mathbf{f}$ is the body force, $p$ is the dynamic pressure, $\mathbf{S}$ is the extra stress tensor. The term $\frac{D \mathbf{V}}{D t}$ denotes the substantial acceleration consisting of the local derivative $\frac{\partial \mathbf{V}}{\partial t}$ and the convective derivative $\nabla . \mathbf{V}$ and $\mathbf{J}$ is the electric current density, $\mathbf{B}$ is the total magnetic field and $\mathbf{B}=\mathbf{B}_{\mathbf{0}}+\mathbf{b}$ (where $\mathbf{B}_{\mathbf{0}}$ represents the imposed magnetic field and $\mathbf{b}$ denotes the induced magnetic field). In the absence of displacement currents, the modified Ohm's law and Maxwell's equations [11,15] are,

$$
\begin{gathered}
\mathbf{J}=\sigma[\mathbf{E}+\mathbf{V} \times \mathbf{B}] . \\
\operatorname{div} \mathbf{B}=0, \quad \nabla \times \mathbf{B}=\mu_{m} \mathbf{J}, \quad \operatorname{cur} l \mathbf{E}=-\frac{\partial B}{\partial t} .
\end{gathered}
$$

where $\sigma$ is the electrical conductivity, $\mathbf{E}$ the electric field and $\mu_{m}$ the magnetic permeability. From Ohm's law and Maxwell's equations an evolution for the magnetic flux B can be obtained easily. This is known as the magnetic induction equation which shows that the motion of an electrically conducting fluid in an applied magnetic field induces a magnetic field in the medium. We assume that the total magnetic field $\mathbf{B}$ is perpendicular to the velocity field $\mathbf{V}$ and the induced magnetic field $\mathbf{b}$ is negligible compared to the applied magnetic field $\mathbf{B}_{\mathbf{0}}$ so that magnetic Reynolds number is small. Since no external electric field is applied, and the effect of polarization of the ionized fluid is negligible, the fluid flow region is assumed to be free of electric field. Under these assumptions, the magneto-hydrodynamics force involved in equation (2) can be put into the form,

$$
\mathbf{J} \times \mathbf{B}=-\sigma \mathbf{B}_{\mathbf{0}}^{2} \mathbf{V}
$$

As discussed in $[5,8]$, the stress tensor defining a Power law fluid is given by:

$$
\begin{gathered}
\mathbf{S}=\mu_{e f f} \mathbf{A}_{\mathbf{1}}, \\
\mu_{e f f}=\eta\left|\sqrt{\frac{\operatorname{tr}\left(\mathbf{A}_{\mathbf{1}}^{\mathbf{2}}\right)}{2}}\right|^{n-1},
\end{gathered}
$$

and where $\eta$ is the coefficient of viscosity and $n$ is the Power law index. The Rivilin-Ericksen tensor, $\mathbf{A}_{\mathbf{1}}$ is defined by:

$$
\mathbf{A}_{1}=\nabla \mathbf{V}+(\nabla \mathbf{V})^{T}
$$

Remark: On behalf of consequent model for $n<1$ the fluid is "pseudoplastic" for model or "shear thinning" for $n>1$ the fluid is "dilatant" or "shear-thickening" and for $n=1$ the Newtonian fluid is recovered.

\section{Formulation of the problem and solution for lifting case}

Consider a container filled with Power law MHD fluid. A wide cylinder moves vertically upward through container with constant velocity $U_{0}$. Since the cylinder moves upward, it picks up a thin fluid film of thickness $\delta$. Due to gravity, the fluid film tends to drain down the cylinder. we choose an $r z-$ coordinate system such that $r$-axis is normal to the cylinder and $z$-axis along the axis of cylinder in upward direction as shown in Figure1. We assume that the cylinder is non-conducting and the magnetic field is applied along the $r$-axis. Assuming that the flow is steady, laminar and uniform and surface tension effects are negligible, the only nonzero velocity component is in $z$-direction. For the reasons mentioned here we assume that,

$$
\mathbf{V}=[0,0, w(r)], \quad \mathbf{S}=S(r) .
$$

Using equation (9), the continuity equation (1) is identically satisfied and by using equation (5) the momentum equation (2) reduces to

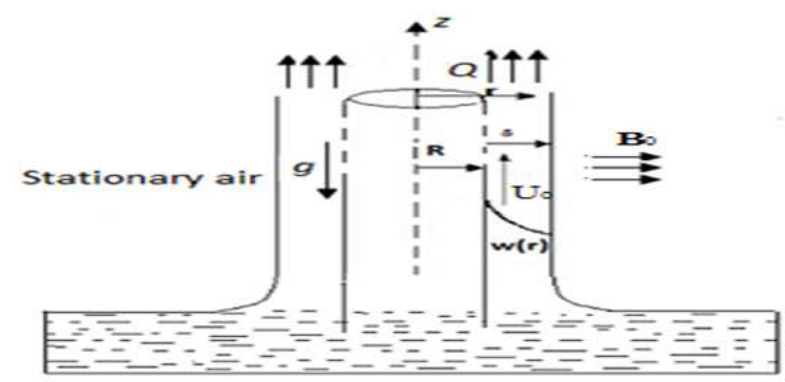

Fig.1. Geometry of the flow of moving cylinder through a power law MHD fluid. 
r-component

$$
0=-\frac{\partial p}{\partial r}
$$

$\theta$-component

$$
0=-\frac{\partial p}{\partial \theta}
$$

Z-component

$$
\frac{\partial p}{\partial z}=\frac{\eta}{r} \frac{\partial}{\partial r}\left(r\left|\frac{\partial w}{\partial r}\right|^{n-1} \frac{\partial w}{\partial r}\right)-\rho g-\sigma B_{0}^{2} w(r) .
$$

Equations (10) and (11) imply that $p=p(z)$ only. Imagine that pressure $p$ is atmospheric pressure i.e., $p$ is zero (gauge pressure) everywhere. As we are discussing the flow problem, we take $\frac{\partial w}{\partial r}$ positive [16]. Thus equation (12) reduces to,

$$
0=\frac{\eta}{r} \frac{d}{d r}\left(r\left(\frac{d w}{d r}\right)^{n}\right)-\rho g-\sigma B_{0}^{2} w(r),
$$

which is a nonlinear differential equation. The associated boundary conditions are:

$$
\begin{aligned}
\frac{d w}{d r}=0 \quad \text { at } & r=R+\delta, \\
w & =U_{0} \quad \text { at } \quad r=R .
\end{aligned}
$$

Introducing dimensionless parameters,

$$
r^{*}=\frac{r}{R}, \quad w^{*}=\frac{w}{U_{0}},
$$

in equation (13) and boundary conditions (14) and (15), we achieve after dropping " $*$ "

$$
\frac{1}{r} \frac{d}{d r}\left(r\left(\frac{d w}{d r}\right)^{n}\right)-\varepsilon w(r)=S_{t},
$$

and associative boundary conditions will be

$$
\begin{aligned}
\frac{d w}{d r}=0 & \text { at } & r=M, \\
w=1 & \text { at } & r=1 .
\end{aligned}
$$

where $S_{t}=\frac{\rho g R^{2}}{\mu_{e f f} U_{0}}$ is the Stoke's number, $\mu_{e f f}=\frac{\eta}{\left(\frac{R}{U_{0}}\right)^{n-1}}$ is power law fluid parameter, $\varepsilon=\frac{\sigma B_{0}^{2} R^{n+1}}{\eta U_{0}^{n}}$ and $M=1+\frac{\delta}{R}$.

\section{Perturbation solution}

We assume $\varepsilon$ be a small parameter and velocity profile $w(r, \varepsilon)$ can be expressed as a power series given by,

$$
w(r, \varepsilon) \approx w_{0}+\varepsilon w_{1}+\varepsilon^{2} w_{2}+\ldots
$$

Using equation (20) into (17) and (18) - (19) and equating like power of $\varepsilon$ we obtain the following set of problems along with their corresponding boundary conditions: zeroth order problem

$$
\varepsilon^{0}: \frac{1}{r} \frac{d}{d r}\left(r\left(\frac{d w_{0}}{d r}\right)^{n}\right)=S_{t},
$$

with boundary conditions,

$$
\begin{array}{cccc}
\frac{d w_{0}}{d r}=0 & \text { at } & r=M, \\
w_{0}=1 & \text { at } & r=1 .
\end{array}
$$

First order problem

$$
\varepsilon^{1}: \frac{1}{r} \frac{d}{d r}\left(r n\left(\frac{d w_{0}}{d r}\right)^{n-1} \frac{d w_{1}}{d r}\right)-w_{0}=0,
$$

with boundary conditions,

$$
\begin{array}{cccc}
\frac{d w_{1}}{d r}=0 & \text { at } & r=M, \\
w_{1}=0 & \text { at } & r=1 .
\end{array}
$$

Here two cases arise:

Case-I: $n=1$ (Newtonian fluid)

Case-II: $n \neq 1$ (Power law fluid)

\subsection{Solution for the Newtonian MHD fluid}

\subsubsection{Velocity Profile}

Zeroth order solution:

The solution of equation (21) by using boundary conditions (22) and (23) is,

$$
w_{0}=1-\frac{S_{t}}{4}\left[\left(1-r^{2}\right)+2 M^{2} \ln r\right] .
$$

First-order solution:

Making use of zeroth order solution (27) into (24) and subject to conditions (25) and (26) is given by,

$$
\begin{aligned}
w_{1} & =\frac{\left(r^{2}-1-2 M^{2} \ln r\right)}{4}-\frac{S_{t}}{64}\left[4\left(1-2 M^{2}\right) r^{2}-r^{4}\right. \\
& -3+8 M^{2}+\left(12 M^{4}-8 M^{2}-16 M^{4} \ln M\right) \ln r \\
& \left.+8 M^{2} r^{2} \ln r\right] .
\end{aligned}
$$

Thus the perturbation solution correct up to first order:

$$
\begin{aligned}
w(r) & =1-\frac{S_{t}}{4}\left[\left(1-r^{2}\right)+2 M^{2} \ln (r)\right] \\
& +\frac{\varepsilon\left(r^{2}-1-2 M^{2} \ln r\right)}{4}-\frac{\varepsilon S_{t}}{64}\left[4\left(1-2 M^{2}\right) r^{2}-r^{4}\right. \\
& -3+8 M^{2}+\left(12 M^{4}-8 M^{2}-16 M^{4} \ln M\right) \ln r \\
& \left.+8 M^{2} r^{2} \ln r\right] .
\end{aligned}
$$

The solution for simple case of Newtonian fluid without MHD effects cab be obtained by putting $\varepsilon=0$ in (29). 


\subsubsection{Volume Flow Rate}

In dimensionless form, the volume flow rate $Q$, is given by,

$$
Q=\int_{0}^{2 \pi} \int_{1}^{M} r w(r) d r d \theta=2 \pi \int_{1}^{M} r w(r) d r .
$$

By making use of equation (29) in equation (30), we obtain,

$$
\begin{aligned}
Q & =\pi\left(M^{2}-1\right)-\frac{S_{t} \pi}{8}\left[4 M^{4} \ln (M)-\left(M^{2}-1\right)^{2}\right. \\
& \left.-2 M^{2}\left(M^{2}-1\right)\right]+\frac{\varepsilon \pi}{8}\left[\left(3 M^{4}-4 M^{2}+1-4 M^{4} \ln M\right)\right. \\
& -\frac{S_{t}}{24}\left(60 M^{4}-34 M^{6}-30 M^{2}+4+72 M^{6} \ln M\right. \\
& \left.\left.-48 M^{4} \ln M-48 M^{6}(\ln M)^{2}\right)\right]
\end{aligned}
$$

\subsubsection{Average velocity}

The average film velocity $\bar{V}$ is then given by,

$$
\bar{V}=\frac{Q}{\pi\left(M^{2}-1\right)},
$$

Using equation (31) in equation (32), we obtain,

$$
\begin{aligned}
\bar{V} & =1-\frac{S_{t}}{8}\left[\frac{4 M^{4} \ln (M)}{\left(M^{2}-1\right)}-3 M^{2}+1\right] \\
& +\frac{\varepsilon}{8\left(M^{2}-1\right)}\left[\left(3 M^{4}-4 M^{2}+1-4 M^{4} \ln M\right)\right. \\
& -\frac{S_{t}}{24}\left(60 M^{4}-34 M^{6}-30 M^{2}+4\right. \\
& \left.\left.+72 M^{6} \ln M-48 M^{4} \ln M-48 M^{6}(\ln M)^{2}\right)\right] .
\end{aligned}
$$

Equation (33) gives the net upward flow of fluid. For $\bar{V}>$ 0 ,

$$
\begin{aligned}
1 & >\frac{S_{t}}{8}\left[\frac{4 M^{4} \ln (M)}{\left(M^{2}-1\right)}-3 M^{2}+1 .\right] \\
& -\frac{\varepsilon}{8\left(M^{2}-1\right)}\left[\left(3 M^{4}-4 M^{2}+1-4 M^{4} \ln M\right)\right. \\
& -\frac{S_{t}}{24}\left(60 M^{4}-34 M^{6}-30 M^{2}+4\right. \\
& \left.\left.+72 M^{6} \ln M-48 M^{4} \ln M-48 M^{6}(\ln M)^{2}\right)\right] .
\end{aligned}
$$

\subsection{Solution for power law MHD fluid}

\subsubsection{Velocity Profile}

Zeroth order solution:

By using binomial series and applying boundary conditions (22) and (23), solution of equation (21) will be,

$$
\begin{aligned}
w_{0} & =1-\left(\frac{S_{t}}{2}\right)^{\frac{1}{n}}\left(\sum _ { k = 0 } ^ { \infty } ( \begin{array} { l } 
{ \frac { 1 } { n } } \\
{ k }
\end{array} ) \frac { ( - 1 ) ^ { k } M ^ { - 2 k + \frac { 2 } { n } } } { 2 k - \frac { 1 } { n } + 1 } \left(r^{2 k-\frac{1}{n}+1}\right.\right. \\
& -1)) .
\end{aligned}
$$

First-order solution:

Making use of zeroth-order solution (35) into (24), after using equations (25) and (26), we obtain,

$$
\begin{aligned}
w_{1}= & \frac{1}{n}\left(\frac{S_{t}}{2}\right)^{\frac{1-n}{n}}\left[\sum_{l=0}^{\infty}\left(\begin{array}{c}
\frac{1-n}{n} \\
l
\end{array}\right) \frac{(-1)^{l} M^{-2 l+\frac{2}{n}-2}}{2}\right. \\
& \left\{\left(\frac{r^{2 l-\frac{1}{n}+3}-1}{2 l-\frac{1}{n}+3}\right)-\frac{M^{2}}{2}\left(\frac{r^{2 l-\frac{1}{n}+1}-1}{2 l-\frac{1}{n}+1}\right)\right\} \\
- & \left(\frac{S_{t}}{2}\right)^{\frac{1}{n}} \sum_{k=0}^{\infty} \sum_{l=0}^{\infty}\left(\begin{array}{c}
\frac{1}{n} \\
k
\end{array}\right)\left(\begin{array}{c}
\frac{1-n}{n} \\
l
\end{array}\right) \frac{(-1)^{l+k} M^{-2 k-2 l+\frac{4}{n}-2}}{2 k-\frac{1}{n}+1} \\
& \left\{\frac{\left(r^{2 k+2 l-\frac{2}{n}+4}-1\right)}{\left(2 k-\frac{1}{n}+3\right)\left(2 k+2 l-\frac{2}{n}+4\right)}-\frac{\left(r^{2 l-\frac{1}{n}+3}-1\right)}{2\left(2 l-\frac{1}{n}+3\right)}\right. \\
- & \frac{M^{2 k-\frac{1}{n}+3}\left(r^{2 l-\frac{1}{n}+1}-1\right)}{\left(2 k-\frac{1}{n}+3\right)\left(2 l-\frac{1}{n}+1\right)} \\
+ & \left.\left.\frac{M^{2}\left(r^{2 l-\frac{1}{n}+1}-1\right)}{2\left(2 l-\frac{1}{n}+1\right)}\right\}\right]
\end{aligned}
$$

Inserting equations $(35,36)$ in to series $(20)$, one get the solution of equation (17) of the form:

$$
\begin{aligned}
w= & 1-\left(\frac{S_{t}}{2}\right)^{\frac{1}{n}}\left(\sum _ { k = 0 } ^ { \infty } ( \begin{array} { l } 
{ \frac { 1 } { n } } \\
{ k }
\end{array} ) \frac { ( - 1 ) ^ { k } M ^ { - 2 k + \frac { 2 } { n } } } { 2 k - \frac { 1 } { n } + 1 } \left(r^{2 k-\frac{1}{n}+1}\right.\right. \\
- & 1))+\frac{\varepsilon}{n}\left(\frac{S_{t}}{2}\right)^{\frac{1-n}{n}}\left[\sum_{l=0}^{\infty}\left(\begin{array}{c}
\frac{1-n}{n} \\
l
\end{array}\right) \frac{(-1)^{l} M^{-2 l+\frac{2}{n}-2}}{2}\right. \\
& \left\{\left(\frac{r^{2 l-\frac{1}{n}+3}-1}{2 l-\frac{1}{n}+3}\right)-\frac{M^{2}}{2}\left(\frac{r^{2 l-\frac{1}{n}+1}-1}{2 l-\frac{1}{n}+1}\right)\right\} \\
- & \left(\frac{S_{t}}{2}\right)^{\frac{1}{n}} \sum_{k=0}^{\infty} \sum_{l=0}^{\infty}\left(\begin{array}{c}
\frac{1}{n} \\
k
\end{array}\right)\left(\begin{array}{c}
1-n \\
l
\end{array}\right) \frac{(-1)^{l+k} M^{-2 k-2 l+\frac{4}{n}-2}}{2 k-\frac{1}{n}+1} \\
& \left\{\frac{\left(r^{2 k+2 l-\frac{2}{n}+4}-1\right)}{\left(2 k-\frac{1}{n}+3\right)\left(2 k+2 l-\frac{2}{n}+4\right)}-\frac{1}{2\left(2 l-\frac{1}{n}+3\right)}\right. \\
- & \left.\left.\frac{M^{2 k-\frac{1}{n}+3}\left(r^{2 l-\frac{1}{n}+1}-1\right)}{\left(2 k-\frac{1}{n}+3\right)\left(2 l-\frac{1}{n}+1\right)}+\frac{M^{2}\left(r^{2 l-\frac{1}{n}+1}-1\right)}{2\left(2 l-\frac{1}{n}+1\right)}\right\}\right](3)
\end{aligned}
$$

\subsubsection{Volume Flow Rate}

By making use of equation (37) in equation (30), we obtain,

$Q=\pi\left(M^{2}-1\right)-2 \pi\left(\frac{S_{t}}{2}\right)^{\frac{1}{n}} \sum_{k=0}^{\infty}\left(\begin{array}{l}\frac{1}{n} \\ k\end{array}\right) \frac{(-1)^{k} M^{-2 k+\frac{2}{n}}}{2 k-\frac{1}{n}+1}$ 


$$
\begin{aligned}
& {\left[\frac{\left(M^{2 k-\frac{1}{n}+3}-1\right)}{2 k-\frac{1}{n}+3}-\frac{\left(M^{2}-1\right)}{2}\right]+\frac{2 \pi \varepsilon}{n}\left(\frac{S_{t}}{2}\right)^{\frac{1-n}{n}} } \\
& {\left[\sum _ { l = 0 } ^ { \infty } ( \begin{array} { c } 
{ \frac { 1 - n } { n } } \\
{ l }
\end{array} ) \frac { ( - 1 ) ^ { l } M ^ { - 2 l + \frac { 2 } { n } - 2 } } { 2 } \left\{\frac{1}{2 l-\frac{1}{n}+3}\right.\right.} \\
& \left(\frac{\left(M^{2 l-\frac{1}{n}+5}-1\right)}{2 l-\frac{1}{n}+5}-\frac{M^{2}-1}{2}\right)-\frac{M^{2}}{2\left(2 l-\frac{1}{n}+1\right)} \\
& \left.\left(\frac{M^{2 l-\frac{1}{n}+3}}{2 l-\frac{1}{n}+3}-\frac{M^{2}-1}{2}\right)\right\}-\left(\frac{S_{t}}{2}\right)^{\frac{1}{n}} \sum_{k=0}^{\infty} \sum_{l=0}^{\infty} \\
& \left(\begin{array}{l}
\frac{1}{n} \\
k
\end{array}\right)\left(\frac{1-n}{n}\right) \frac{(-1)^{l+k} M^{-2 k-2 l+\frac{4}{n}-2}}{2 k-\frac{1}{n}+1} \\
& \left\{\frac { 1 } { ( 2 k - \frac { 1 } { n } + 3 ) ( 2 k + 2 l - \frac { 2 } { n } + 4 ) } \left(\frac{M^{2 k+2 l-\frac{2}{n}+6}-1}{2 k+2 l-\frac{2}{n}+6}\right.\right. \\
- & \left.\frac{M^{2}-1}{2}\right)-\frac{M^{2 k-\frac{1}{n}+3}}{\left(2 k-\frac{1}{n}+3\right)\left(2 l-\frac{1}{n}+1\right)}\left(\frac{M^{2 l-\frac{1}{n}+3}-1}{2 l-\frac{1}{n}+3}\right. \\
- & \left.\frac{M^{2}-1}{2}\right)-\frac{1}{2\left(2 l-\frac{1}{n}+3\right)}\left(\frac{M^{2 l-\frac{1}{n}+5}-1}{2 l-\frac{1}{n}+5}-\frac{M^{2}-1}{2}\right) \\
+ & \left.\left.\frac{M^{2}}{2\left(2 l-\frac{1}{n}+1\right)}\left(\frac{M^{2 l-\frac{1}{n}+3}-1}{2 l-\frac{1}{n}+3}-\frac{M^{2}-1}{2}\right)\right\}\right] .
\end{aligned}
$$

\subsubsection{Average Velocity}

By using equation (38) in equation (32) we obtain,

$$
\begin{aligned}
\bar{V}= & 1-2\left(\frac{S_{t}}{2}\right)^{\frac{1}{n}} \sum_{k=0}^{\infty}\left(\begin{array}{l}
\frac{1}{n} \\
k
\end{array}\right) \frac{(-1)^{k} M^{-2 k+\frac{2}{n}}}{2 k-\frac{1}{n}+1} \\
& {\left[\frac{\left(M^{2 k-\frac{1}{n}+3}-1\right)}{\left(2 k-\frac{1}{n}+3\right)\left(M^{2}-1\right)}-\frac{1}{2}\right]+\frac{2 \varepsilon}{n}\left(\frac{S_{t}}{2}\right)^{\frac{1-n}{n}} } \\
& {\left[\sum _ { l = 0 } ^ { \infty } ( \begin{array} { l } 
{ \frac { 1 - n } { n } } \\
{ l }
\end{array} ) \frac { ( - 1 ) ^ { l } M ^ { - 2 l + \frac { 2 } { n } - 2 } } { 2 } \left\{\frac{1}{2 l-\frac{1}{n}+3}\right.\right.} \\
& \left(\frac{\left(M^{2 l-\frac{1}{n}+5}-1\right)}{\left(2 l-\frac{1}{n}+5\right)\left(M^{2}-1\right)}-\frac{1}{2}\right)-\frac{M^{2}}{2\left(2 l-\frac{1}{n}+1\right)} \\
& \left.\left(\frac{M^{2 l-\frac{1}{n}+3}-1}{\left(M^{2}-1\right)\left(2 l-\frac{1}{n}+3\right)}-\frac{1}{2}\right)\right\}-\left(\frac{S_{t}}{2}\right)^{\frac{1}{n}} \sum_{k=0}^{\infty} \sum_{l=0}^{\infty} \\
& \left(\begin{array}{l}
\frac{1}{n} \\
k
\end{array}\right)\left(\begin{array}{l}
\frac{1-n}{n} \\
l
\end{array}\right) \frac{(-1)^{l+k} M^{-2 k-2 l+\frac{4}{n}-2}}{2 k-\frac{1}{n}+1} \\
& \left\{\frac{1}{\left(2 k-\frac{1}{n}+3\right)\left(2 k+2 l-\frac{2}{n}+4\right)}\right. \\
& \left(\frac{M^{2 k+2 l-\frac{2}{n}+6}-1}{\left(M^{2}-1\right)\left(2 k+2 l-\frac{2}{n}+6\right)}-\frac{1}{2}\right)
\end{aligned}
$$

$$
\begin{aligned}
& -\frac{M^{2 k-\frac{1}{n}+3}}{\left(2 k-\frac{1}{n}+3\right)\left(2 l-\frac{1}{n}+1\right)}\left(\frac{M^{2 l-\frac{1}{n}+3}-1}{\left(M^{2}-1\right)\left(2 l-\frac{1}{n}+3\right)}\right. \\
& \left.-\frac{1}{2}\right)-\frac{1}{2\left(2 l-\frac{1}{n}+3\right)}\left(\frac{M^{2 l-\frac{1}{n}+5}-1}{\left(M^{2}-1\right)\left(2 l-\frac{1}{n}+5\right)}-\frac{1}{2}\right) \\
& \left.\left.+\frac{M^{2}}{2\left(2 l-\frac{1}{n}+1\right)}\left(\frac{M^{2 l-\frac{1}{n}+3}-1}{\left(M^{2}-1\right)\left(2 l-\frac{1}{n}+3\right)}-\frac{1}{2}\right)\right\}\right]
\end{aligned}
$$

Equation (39) gives the net upward flow of fluid. For $\bar{V}>$ 0 ,

$$
\begin{aligned}
& 1>2\left(\frac{S_{t}}{2}\right)^{\frac{1}{n}} \sum_{k=0}^{\infty}\left(\begin{array}{l}
\frac{1}{n} \\
k
\end{array}\right) \frac{(-1)^{k} M^{-2 k+\frac{2}{n}}}{2 k-\frac{1}{n}+1} \\
& {\left[\frac{\left(M^{2 k-\frac{1}{n}+3}-1\right)}{\left(2 k-\frac{1}{n}+3\right)\left(M^{2}-1\right)}-\frac{1}{2}\right]+\frac{2 \varepsilon}{n}\left(\frac{S_{t}}{2}\right)^{\frac{1-n}{n}}} \\
& {\left[\sum _ { l = 0 } ^ { \infty } ( \begin{array} { c } 
{ \frac { 1 - n } { n } } \\
{ l }
\end{array} ) \frac { ( - 1 ) ^ { l } M ^ { - 2 l + \frac { 2 } { n } - 2 } } { 2 } \left\{\frac{1}{2 l-\frac{1}{n}+3}\right.\right.} \\
& \left(\frac{\left(M^{2 l-\frac{1}{n}+5}-1\right)}{\left(2 l-\frac{1}{n}+5\right)\left(M^{2}-1\right)}-\frac{1}{2}\right)-\frac{M^{2}}{2\left(2 l-\frac{1}{n}+1\right)} \\
& \left.\left(\frac{M^{2 l-\frac{1}{n}+3}-1}{\left(M^{2}-1\right)\left(2 l-\frac{1}{n}+3\right)}-\frac{1}{2}\right)\right\}-\left(\frac{S_{t}}{2}\right)^{\frac{1}{n}} \sum_{k=0}^{\infty} \sum_{l=0}^{\infty} \\
& \left(\begin{array}{c}
\frac{1}{n} \\
k
\end{array}\right)\left(\begin{array}{c}
\frac{1-n}{n} \\
l
\end{array}\right) \frac{(-1)^{l+k} M^{-2 k-2 l+\frac{4}{n}-2}}{2 k-\frac{1}{n}+1} \\
& \left\{\frac{1}{\left(2 k-\frac{1}{n}+3\right)\left(2 k+2 l-\frac{2}{n}+4\right)}\right. \\
& \left(\frac{M^{2 k+2 l-\frac{2}{n}+6}-1}{\left(M^{2}-1\right)\left(2 k+2 l-\frac{2}{n}+6\right)}-\frac{1}{2}\right) \\
& -\frac{M^{2 k-\frac{1}{n}+3}}{\left(2 k-\frac{1}{n}+3\right)\left(2 l-\frac{1}{n}+1\right)}\left(\frac{M^{2 l-\frac{1}{n}+3}-1}{\left(M^{2}-1\right)\left(2 l-\frac{1}{n}+3\right)}\right. \\
& \left.-\frac{1}{2}\right)-\frac{1}{2\left(2 l-\frac{1}{n}+3\right)}\left(\frac{M^{2 l-\frac{1}{n}+5}-1}{\left(M^{2}-1\right)\left(2 l-\frac{1}{n}+5\right)}-\frac{1}{2}\right) \\
& \left.\left.+\frac{M^{2}}{2\left(2 l-\frac{1}{n}+1\right)}\left(\frac{M^{2 l-\frac{1}{n}+3}-1}{\left(M^{2}-1\right)\left(2 l-\frac{1}{n}+3\right)}-\frac{1}{2}\right)\right\}\right] \text {. }
\end{aligned}
$$

\section{Solution for drainage case}

Consider steady, parallel, laminar flow of an incompressible Power law MHD fluid down an infinite vertical cylinder. As a result, a thin uniform fluid film of thickness $\delta$ is formed in contact with stationary air. The geometry of the problem is shown in Figure2. We choose an $r z$-coordinate system such that $r$-axis is normal to cylinder and $z$-axis along the cylinder axis in downward direction. We assume that the fluid is non-conducting and the magnetic field is applied along the $r$-axis, there is no 
applied (force) pressure driving the flow and fluid falls under the action of gravity, so the governing equation equation (17) becomes,

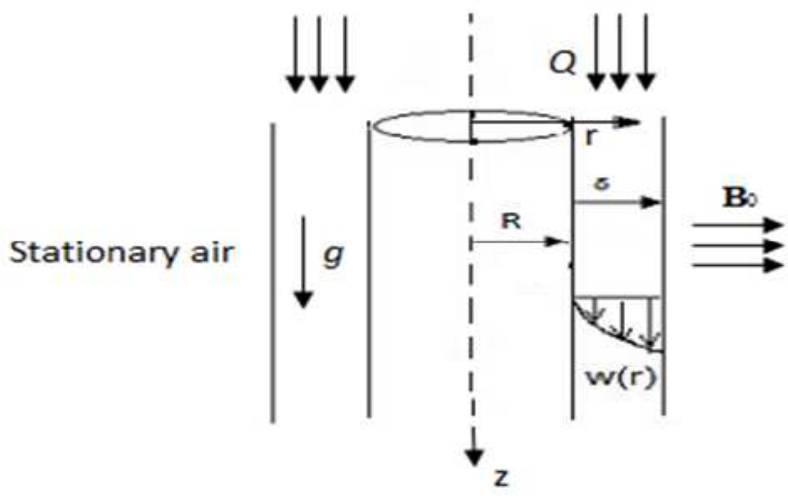

Fig.2. Geometry of the thin film flow down a vertical cylinder.

$$
\begin{aligned}
& \frac{1}{r} \frac{d}{d r}\left(r\left(\frac{d w}{d r}\right)^{n}\right)-\varepsilon w(r)=-S_{t} \\
\frac{d w}{d r}=0 & \text { at } \quad r=M . \\
w=0 & \text { at } \quad r=1,
\end{aligned}
$$

Using Perturbation series method to this problem, we get, different problems each corresponding to different order of $\varepsilon$.

\section{zeroth order problem}

$$
\varepsilon^{0}: \frac{1}{r} \frac{d}{d r}\left(r\left(\frac{d w_{0}}{d r}\right)^{n}\right)=-S_{t}
$$

with boundary condition,

$$
\begin{array}{rll}
\frac{d w_{0}}{d r}=0 & \text { at } & r=M . \\
w_{0}=0 & \text { at } & r=1,
\end{array}
$$

\section{First order problem}

$$
\varepsilon^{1}: \frac{1}{r} \frac{d}{d r}\left(r n\left(\frac{d w_{0}}{d r}\right)^{n-1} \frac{d w_{1}}{d r}\right)-w_{0}=0
$$

with boundary conditions,

$$
\begin{array}{rll}
\frac{d w_{1}}{d r}=0 & \text { at } & r=M . \\
w_{1}=0 & \text { at } & r=1,
\end{array}
$$

Here two cases arise:

Case-I: $n=1$ (Newtonian fluid)

Case-II: $n \neq 1$ (Power law fluid)

\subsection{Solution for Newtonian MHD fluid}

\subsubsection{Velocity Profile}

Zeroth order solution:

The solution of equation (44) after application of boundary conditions (45) and (46) is,

$$
w_{0}=\frac{S_{t}}{4}\left[\left(1-r^{2}\right)+2 M^{2} \ln r\right]
$$

First-order solution:

Introducing zeroth order solution (50), into (47) and subject to the boundary conditions (48) and (49) the first order solution is given by:

$$
\begin{aligned}
w_{1} & =\frac{S_{t}}{64}\left[4\left(1-2 M^{2}\right) r^{2}-r^{4}-3+8 M^{2}+\left(12 M^{4}\right.\right. \\
& \left.\left.-8 M^{2}-16 M^{4} \ln M\right) \ln r+8 M^{2} r^{2} \ln r\right] .
\end{aligned}
$$

Thus the perturbation solution correct up to first order in $\varepsilon$ is given by,

$$
\begin{aligned}
w(r)= & \frac{S_{t}}{4}\left[\left(1-r^{2}\right)+2 M^{2} \ln r\right]+\frac{\varepsilon S_{t}}{64} \\
& {\left[4\left(1-2 M^{2}\right) r^{2}-r^{4}-3+8 M^{2}+\left(12 M^{4}\right.\right.} \\
- & \left.\left.8 M^{2}-16 M^{4} \ln M\right) \ln r+8 M^{2} r^{2} \ln r\right] .
\end{aligned}
$$

\subsubsection{Volume Flow Rate}

By making use of equation (52) in equation (30), we obtain,

$$
\begin{aligned}
Q & =\frac{S_{t} \pi}{8}\left[4 M^{4} \ln (M)-\left(M^{2}-1\right)^{2}-2 M^{2}\left(M^{2}-1\right)\right] \\
& +\frac{S_{t} \pi \varepsilon}{192}\left[60 M^{4}-34 M^{6}-30 M^{2}+4+72 M^{6} \ln M\right. \\
& \left.-48 M^{4} \ln M-48 M^{6}(\ln M)^{2}\right] .
\end{aligned}
$$

\subsubsection{Average Velocity}

Using equation (53) in equation (32), we obtain

$$
\begin{aligned}
\bar{V} & =\frac{S_{t}}{8}\left[\frac{4 M^{4} \ln (M)}{\left(M^{2}-1\right)}-3 M^{2}+1\right] \\
& +\frac{S_{t} \varepsilon}{192\left(M^{2}-1\right)}\left[60 M^{4}-34 M^{6}-30 M^{2}+4\right. \\
& \left.+72 M^{6} \ln M-48 M^{4} \ln M-48 M^{6}(\ln M)^{2}\right] .
\end{aligned}
$$

\subsection{Solution for power law MHD fluid}

\subsubsection{Velocity Profile}

Zeroth order solution:

By using binomial series and applying boundary 
conditions (45) and (46), solution of equation (44) will be,

$$
w_{0}=\left(\frac{S_{t}}{2}\right)^{\frac{1}{n}}\left(\sum_{k=0}^{\infty}\left(\begin{array}{c}
\frac{1}{n} \\
k
\end{array}\right) \frac{(-1)^{k} M^{-2 k+\frac{2}{n}}}{2 k-\frac{1}{n}+1}\left(r^{2 k-\frac{1}{n}+1}-1\right)\right) .
$$

First-order solution:

Introducing the zeroth-order solution (55) into (47) and solving for first order solution, we obtain,

$$
\begin{aligned}
w_{1}= & \frac{1}{n}\left(\frac{S_{t}}{2}\right)^{\frac{2}{n}-1}\left[\sum_{k=0}^{\infty} \sum_{l=0}^{\infty}\left(\begin{array}{c}
\frac{1}{n} \\
k
\end{array}\right)\left(\begin{array}{c}
\frac{1-n}{n} \\
l
\end{array}\right) \frac{(-1)^{l+k} M^{-2 k-2 l+\frac{4}{n}-2}}{2 k-\frac{1}{n}+1}\right. \\
& \left\{\frac{\left(r^{2 k+2 l-\frac{2}{n}+4}-1\right)}{\left(2 k-\frac{1}{n}+3\right)\left(2 k+2 l-\frac{2}{n}+4\right)}-\frac{\left(r^{2 l-\frac{1}{n}+3}-1\right)}{2\left(2 l-\frac{1}{n}+3\right)}\right. \\
- & \left.\left.\frac{M^{2 k-\frac{1}{n}+3}\left(r^{2 l-\frac{1}{n}+1}-1\right)}{\left(2 k-\frac{1}{n}+3\right)\left(2 l-\frac{1}{n}+1\right)}+\frac{M^{2}\left(r^{2 l-\frac{1}{n}+1}-1\right)}{2\left(2 l-\frac{1}{n}+1\right)}\right\}\right]
\end{aligned}
$$

\subsubsection{Average Velocity}

Using equation (58) in equation (32), we obtain,

$$
\begin{aligned}
\bar{V} & =2\left(\frac{S_{t}}{2}\right)^{\frac{1}{n}} \sum_{k=0}^{\infty}\left(\begin{array}{c}
\frac{1}{n} \\
k
\end{array}\right) \frac{(-1)^{k} M^{-2 k+\frac{2}{n}}}{2 k-\frac{1}{n}+1}\left[\frac{\left(M^{2 k-\frac{1}{n}+3}\right)}{\left(2 k-\frac{1}{n}+3\right)\left(M^{2}-1\right)}\right. \\
- & \left.\frac{1}{2}\right]+\frac{2 \varepsilon}{n}\left(\frac{S_{t}}{2}\right)^{\frac{2}{n}-1}\left[\sum_{k=0}^{\infty} \sum_{l=0}^{\infty}\left(\begin{array}{c}
\frac{1}{n} \\
k
\end{array}\right)\left(\begin{array}{c}
\frac{1-n}{n} \\
l
\end{array}\right)\right. \\
& \frac{(-1)^{l+k} M^{-2 k-2 l+\frac{4}{n}-2}}{2 k-\frac{1}{n}+1}\left\{\frac{1}{\left(2 k-\frac{1}{n}+3\right)\left(2 k+2 l-\frac{2}{n}+4\right)}\right. \\
- & \left.\frac{M^{2 k+2 l-\frac{2}{n}+6}-1}{\left(M^{2}-1\right)\left(2 k+2 l-\frac{2}{n}+6\right)}-\frac{1}{2}\right) \\
- & \left.\frac{1}{2}\right)-\frac{M^{2 k-\frac{1}{n}+3}}{2\left(2 l-\frac{1}{n}+3\right)}\left(\frac{M^{2 l-\frac{1}{n}+3}-1}{\left(M^{2}-1\right)\left(2 l-\frac{1}{n}+5\right)}\right. \\
- & \left.\frac{1}{2}\right)+\frac{\left.M^{2}-1\right)\left(2 l-\frac{1}{n}+3\right)}{2\left(2 l-\frac{1}{n}+1\right)}\left(\frac{M^{2 l-\frac{1}{n}+3}-1}{\left(M^{2}-1\right)\left(2 l-\frac{1}{n}+3\right)}\right. \\
- & \left.\left.\left.\frac{1}{2}\right)\right\} \cdot \frac{1}{n}+1\right)
\end{aligned}
$$

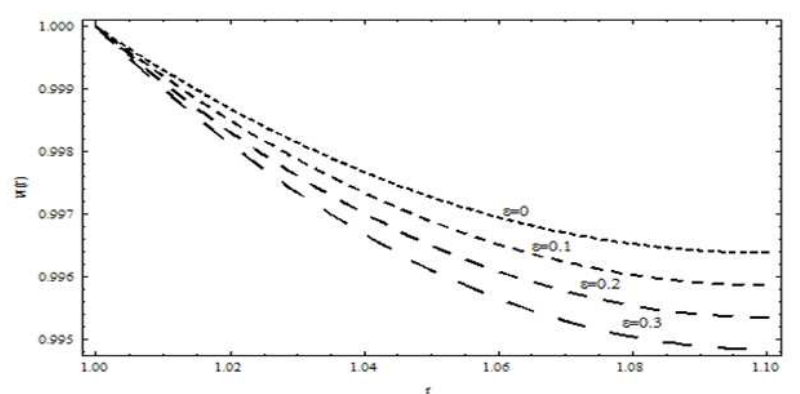

Fig.3. Effect of $\varepsilon$ on velocity profile for Newtonian MHD fluid for lift in thin film flow, when $S_{t}=0.7, M=1.1$. 


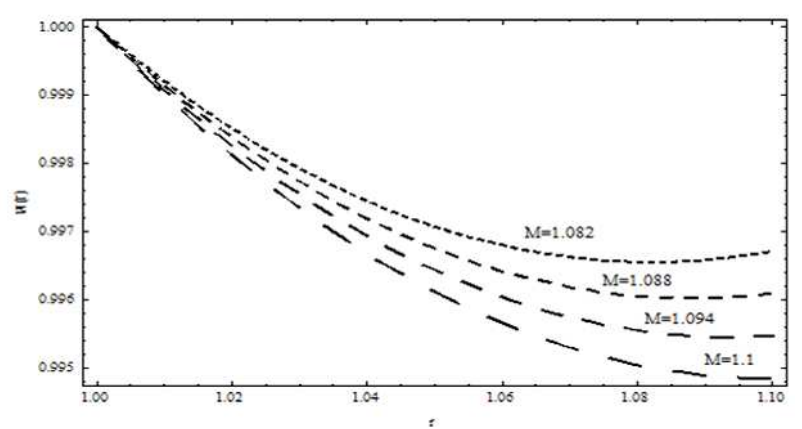

Fig.4. Effect of $M$ on velocity profile for Newtonian MHD fluid for lift in thin film flow, when $\varepsilon=0.3, S_{t}=0.7$.

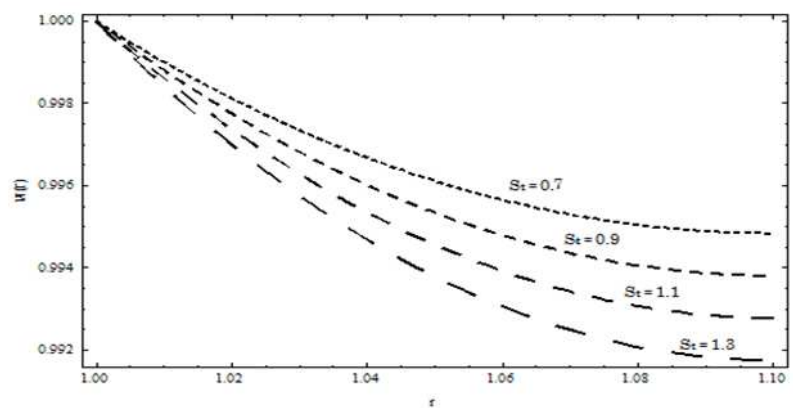

Fig.5. Effect of $S_{t}$ on velocity profile for Newtonian MHD fluid for lift in thin film flow, when $\varepsilon=0.3, M=1.1$.

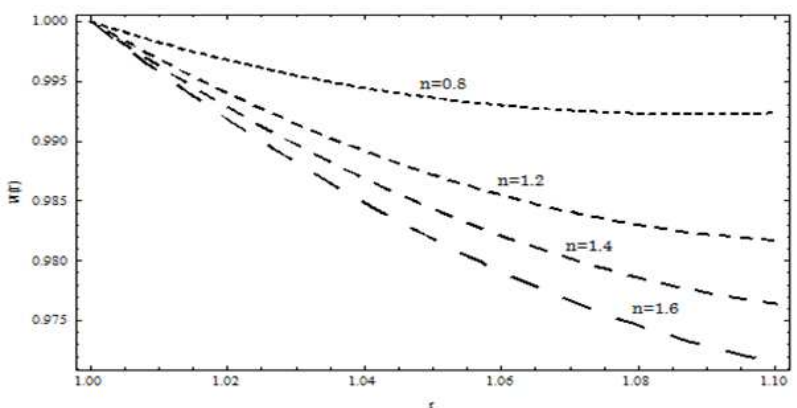

Fig.6. Effect of $n$ on velocity profile for Power law MHD fluid for lift in thin film flow, when $\varepsilon=0.005, S_{t}=2.5, M=1.1$.

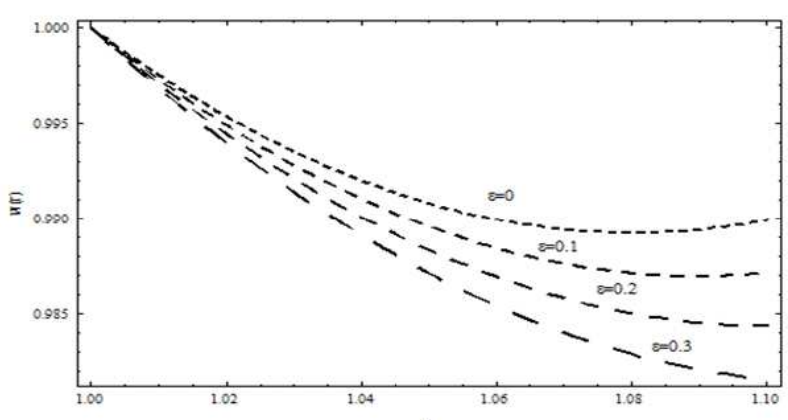

Fig.7. Effect of $\varepsilon$ on velocity profile for Power law MHD fluid for lift in thin film flow, when $n=1.2, S_{t}=2.5, M=1.1$.

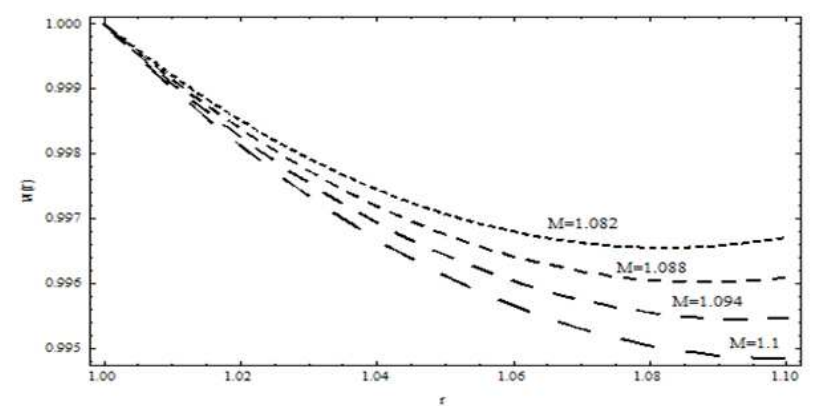

Fig.8. Effect of $M$ on velocity profile for Power law MHD fluid for lift in thin film flow, when $\varepsilon=0.005, S_{t}=2.5, n=1.2$.

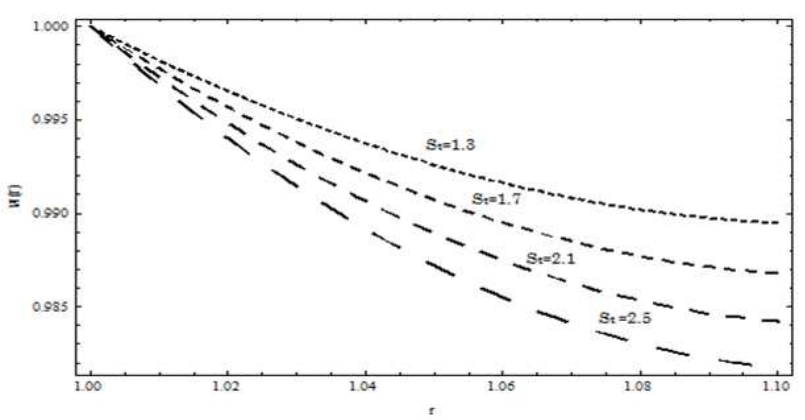

Fig.9. Effect of $S_{t}$ on velocity profile for Power law MHD fluid for lift in thin film flow, when $\varepsilon=0.005, n=1.2, M=1.1$.

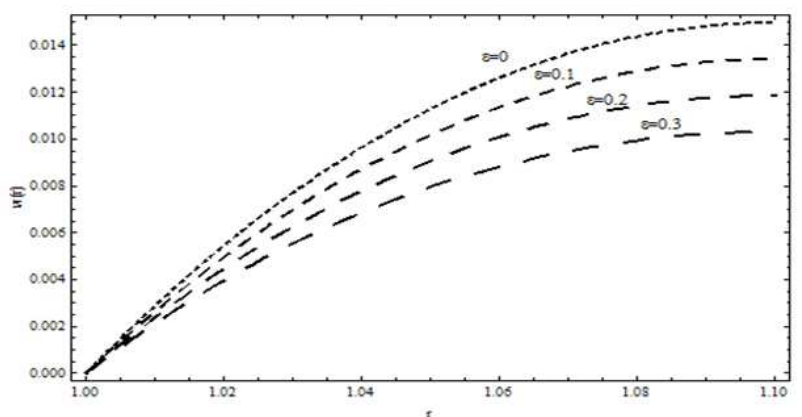

Fig.10. The effect of $\varepsilon$ on velocity profile for Newtonian MHD fluid for drainage in thin film flow, when $S_{t}=2.9, M=1.1 A$.

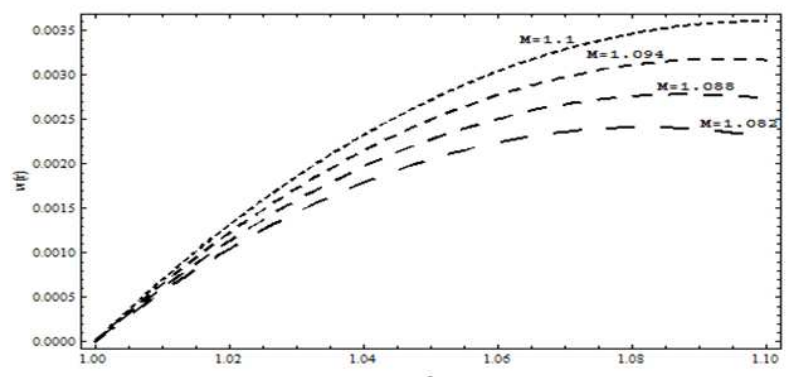

Fig.11. The effect of $M$ on velocity profile for Newtonian MHD fluid for drainage in thin film flow, when $\varepsilon=0.3, S_{t}=0.7$. 


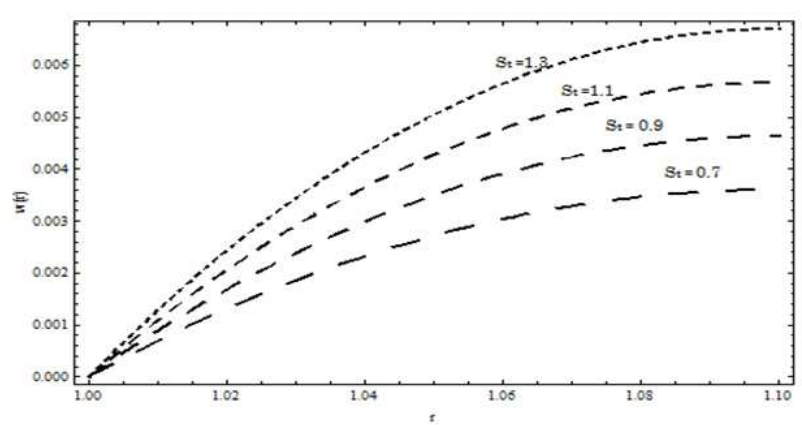

Fig.12. The effect of $S_{t}$ on velocity profile for Newtonian MHD fluid for drainage in thin film flow. when $\varepsilon=0.3, M=1.1$.

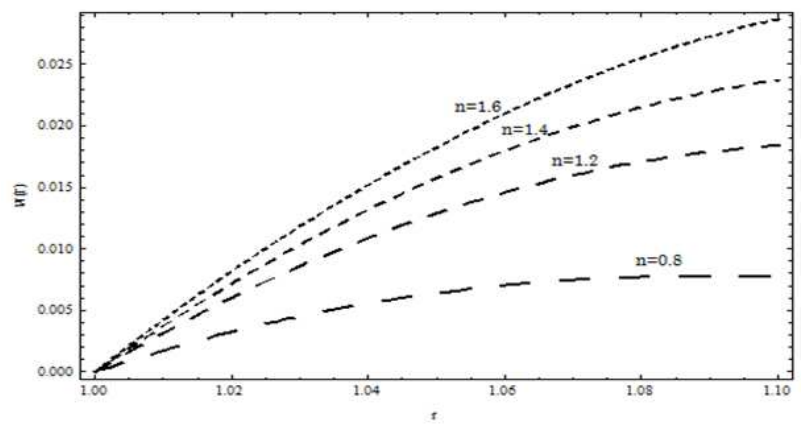

Fig.13. The effect of $n$ on velocity profile for Power law MHD fluid for drainage in thin film flow, when $\varepsilon=0.005, S_{t}=2.5, M=1.1$.

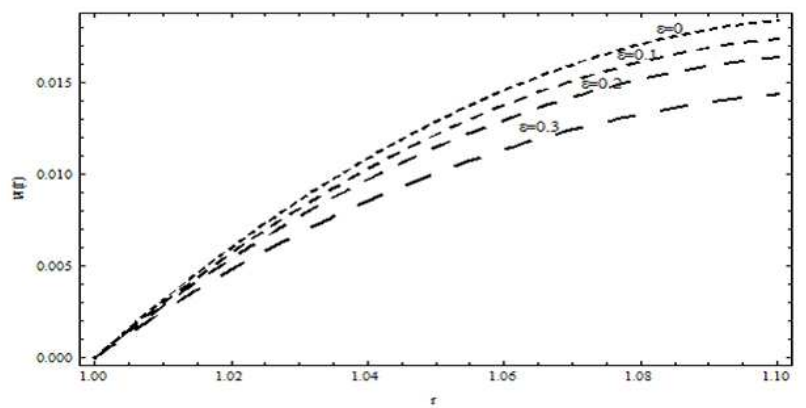

Fig.14. The effect of $\varepsilon$ on velocity profile for Power law MHD fluid for drainage in thin film flow, when $n=1.2, S_{t}=2.5, M=1.1$.

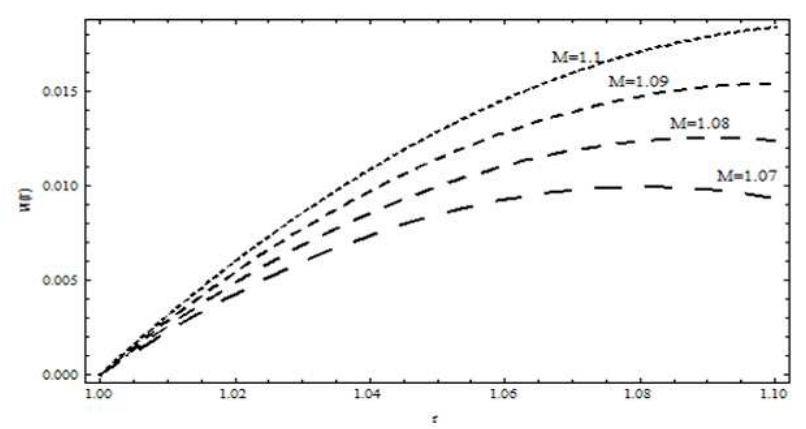

Fig.15. The effect of $M$ on velocity profile for Power law MHD fluid for drainage in thin film flow, when $\varepsilon=0.005, S_{t}=2.5, n=1.2$.

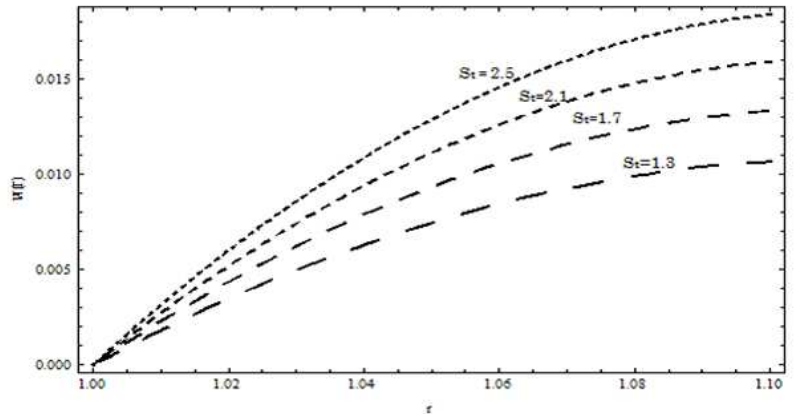

Fig.16. The effect of $S_{t}$ on velocity profile for Power law MHD fluid for drainage in thin film flow, when $\varepsilon=0.005, n=1.2, M=1.1$.

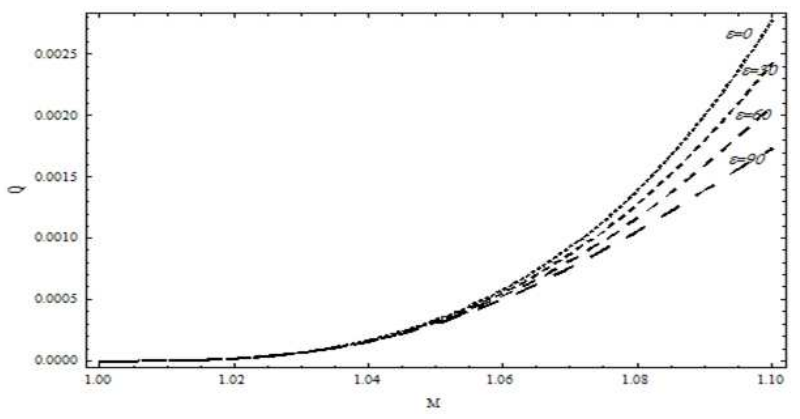

Fig.17. The effect of $\varepsilon$ on flow rate for Newtonian MHD fluid for drainage in thin film flow, when $S_{t}=1.2$

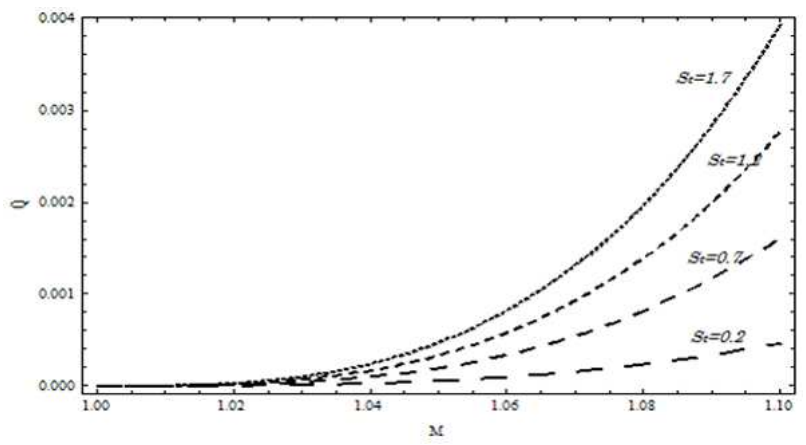

Fig.18. The effect of $S_{t}$ on flow rate for Newtonian MHD fluid for drainage in thin film flow, when $\varepsilon=0.001$.

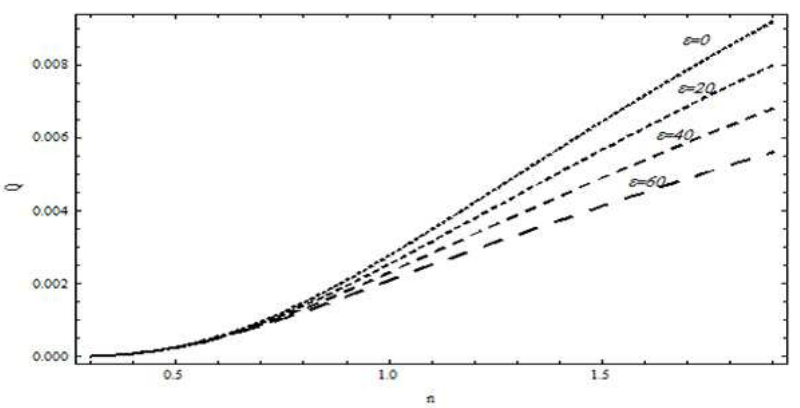

Fig.19. The effect of $\varepsilon$ on flow rate for Power law MHD fluid for drainage in thin film flow, when

$$
S_{t}=1.2, M=1.1 \text {. }
$$




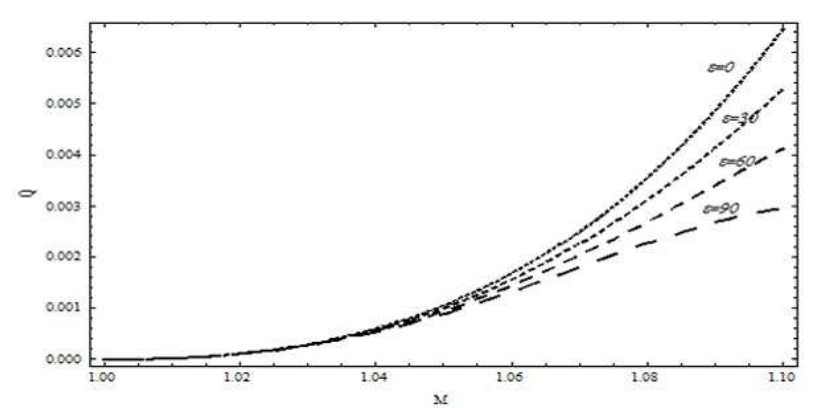

Fig.20. The effect of $\varepsilon$ on flow rate for Power law MHD fluid for drainage in thin film flow, when $S_{t}=1.2, n=1.1$.

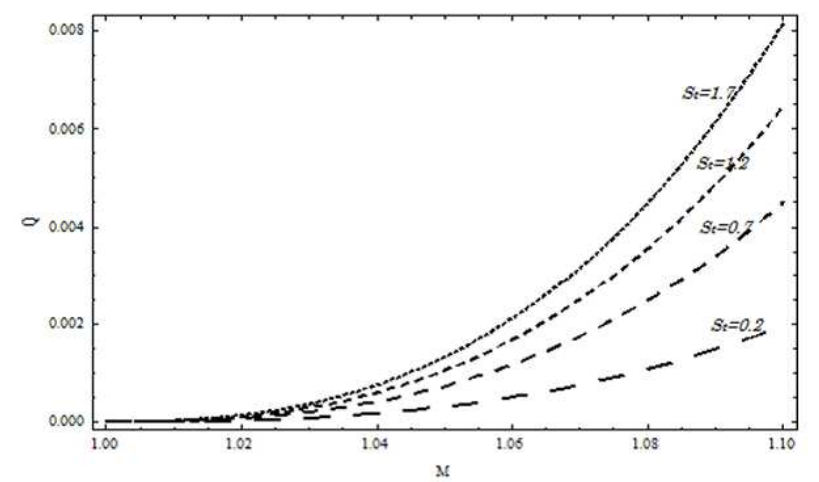

Fig.21. The effect of $S_{t}$ on flow rate for Power law MHD fluid for drainage in thin film flow, when

$$
\varepsilon=0.001, n=1.5 \text {. }
$$

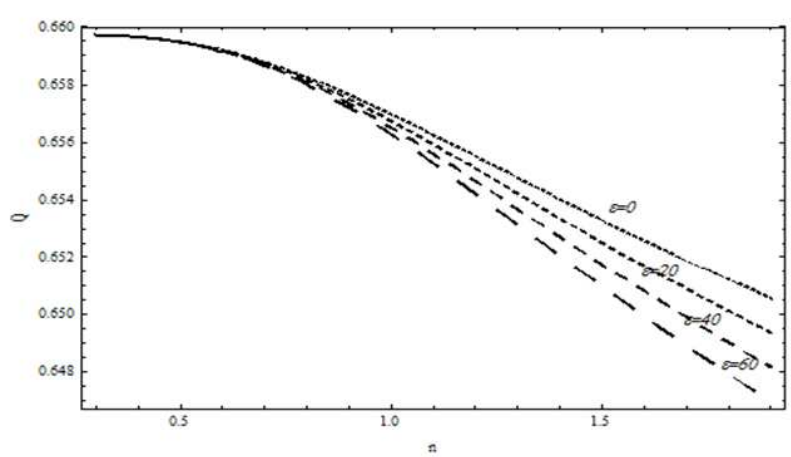

Fig.22. The effect of $\varepsilon$ on flow rate for Power law MHD fluid for lift in thin film flow, when $S_{t}=1.2, M=1.1$.

\section{Results AND Discussion}

The dependence of flow quantities under the value of Power law index $n$, magnetic parameter $\varepsilon$, parameter $M$ and Stokes' number $S_{t}$ are observed physically through figures (3) - (21). The variation of axial velocity for $n, \varepsilon, M$ and $S_{t}$ for both Newtonian and Power law MHD fluid in case of lift is displayed in figures (3) - (9). In figures (3) - (9), we observed that, with an increase in $n, \varepsilon, M$ and $S_{t}$, velocity profile decreases. The difference of $n, \varepsilon, M$ and $S_{t}$ for drainage of fluid film in figure (10) (16) have been plotted, in which it is observed that velocity of fluid film increase for all significant changes in the flow parameters but decreases for magnetic parameter. Dissimilarity is also observed for $n, \varepsilon, M$ and $S_{t}$ for flow rate of Newtonian and Power law fluid in figures (17) - (22), in which we observed that flow rate increases for all significant parameters without the $\varepsilon$.

\section{Concludung Remarks}

We have presented results for the thin film flow field of a fluid called the Power law MHD fluid, on a vertical cylinder for lift and drainage problem. The resulting nonlinear differential equation has been solved by Perturbation method, which is affective and reliable method for the proposed problem. The velocity profile, flow rate and average velocity have been derived analytically.

\section{References}

[1] N. S. Deshpande, M. Barigou, Vibrational flow of nonNewtonian fluids, Chemical Engineering Science, 56, 3845-3853 (2001).

[2] M. Kemiha, X. Frank, S. Poncin, H. Z. Li, Origin of the negative wake behind a bubble rising in non-Newtonian fluids, Chemical Engineering Science, 61, 4041-4047 (2006).

[3] Jie Chen, Xi-Yun Lu, Numerical investigation of the nonNewtonian pulsatile blood flow in a bifurcation model with non-planar branch, Journal of Biomechanics, 39, 818-832 (2006).

[4] F. M. Mahomed, T. Hayat, E. Momoniat, S. Asghar, Gliding motion of bacterium in a non-Newtonian slime, Nonlinear Analysis: Real World Applications, 8, 853-864 (2007).

[5] Yong-Li Chen, Xu-Dong Cao,Ke-Qin Zhu, A gray lattice Boltzmann model for power-law fluid and its application in the study of slip velocity at porous interface, J. NonNewtonian Fluid Mech., 159, 130-136 (2009).

[6] J. N. Kapur, Flows of Power-Law Fluid Past a Flat Plate with uniform suction and between two parallel plates with uniform suction and injection, Journal of the physical society of Japan, 18, 578-581 (1963).

[7] Amir Nejat, Alireza Jalali, Mahkame Sharbatdar, A Newton-Krylov finite volume algorithm for the powerlaw non-Newtonian fluid flow using pseudocompressibility technique, Journal of Non-Newtonian Fluid Mechanics, 166, 1158-1172 (2011),

[8] M. H .R.Ghoreishy and M.Razavi Nouri, A Threedimensional Finite Element Analysis of a Power-law Fluid in an Extrusion Die, Iranian Polymer Journal, 7, (1998).

[9] A. M. Siddiqui and R. Mahmood and Q. K. Ghori. Homotopy perturbation method for thin film flow of a fourth grade fluid down a vertical cylinder, Phys. Lett., A 352, 404-410 (2006) .

[10] T. Hayat and M. Sajid. On analytic solution for thin film flow of a fourth grade fluid down a vertical cylinder, Physics Letters, A 361, 316-322 (2007). 
[11] Mohyuddin MR, Gotz T. Resonance behavior of viscoelastic fluid in Poiseuille flow in the presence of a transversal magnetic field. International Journal for numerical methods in fluids, 49, 837-847 (2005).

[12] Rossow VJ. On flow of electrically conducting fluids over a flat plate in the presence of a transverse magnetic field, NASA, Report no. 1358, 489, (1958).

[13] Pop I., Kumari M., Nath G. Conjugate MHD flow past a flat plate, Acta Mech., 106, 215-220 (1994).

[14] Abel S., Veena PH., Rajagopal KR., Prayin VK. NonNewtonian magnetohydro- dynamic flow over a stretching surface with heat and mass transfer, Int. J. Non-Linear Mech., 39, 1067-1078 (2004).

[15] T. C Papanastasiou, G. C Georgin, Andreas N. Alexandrou, CRC Press Boca Raton London Newyork Washington, D.C, (2000).

[16] Dipak Kumar Dutta. Draining of power law liquid down a vertical plate in the presence of a transverse magnetic field, Indian journal of theoretical physics, 21, 15-24 (1973).

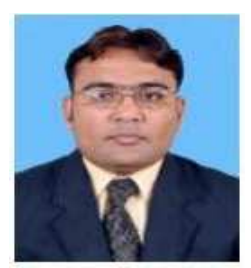

\section{K. N. Memon}

received the MS degree in Applied Mathematics from COMSATS Islamabad, Pakistan. His research interests are in the areas of applied mathematics, especially Newtonian and non-Newtonian fluid mechanics and bio mathematics. He has published research articles in reputed international journals of mathematical and engineering sciences.

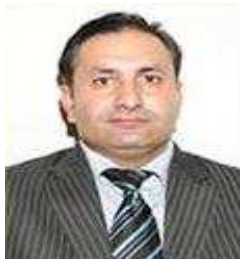

Saeed Islam $(\mathrm{PhD})$, works as Associate Professor and Chairman, the Department of Mathematics, Abdul Wali Khan University Mardan, Pakistan. His research interest is the modeling and simulation using linear and nonlinear differential equations. He has more than 150 research articles in reputed journals listed in JCR and Thomson Master List. He supervised $5 \mathrm{PhDs}$ in COMSATS, Islamabad and Islamia College University Peshawar. He has been supervising more than 15 students of MPhil and PhDs. For his valuable research contribution, the government of Pakistan awarded him the Quaid-i-Azam Gold medal.

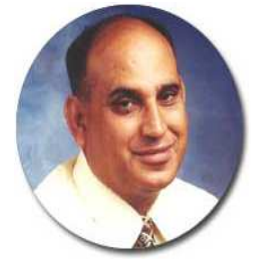

A. M. Siddiqui (PhD), Is Professor of Pennsylvania State University, his research interests are in the areas of applied mathematics, especially Newtonian, non-Newtonian fluid mechanics and bio mathematics including. $\mathrm{He}$ is the renowned mathematician produces many Mphil and $\mathrm{PhD}$ students. He has a great research contribution in the form of documented work in reputed international journals of mathematical and engineering sciences.

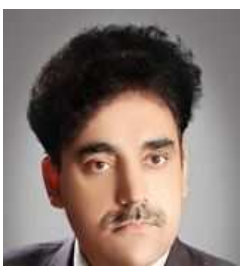

Sher Afzal Khan (PhD), works as an Associate Professor and Chairman, the Department of Computer Sciences, Abdul Wali Khan University Mardan. His research interest is the modeling and simulation using differential equations, fuzzy logic, Petri net and Z. He has many research articles in reputed journals listed in JCR. $\mathrm{He}$ is supervising 6 students of MPhil and PhDs. He is the chairman of the Virtual Foundation for Advancement of Science and Technology (http://vfast.org/), editor in chief of VAWKUM transactions on Computer Sciences (http://vfast.org/index.php/VTCS). Currently, he is the conference chair of the International Conference on Computational and Social Sciences (ICCSS-2013) (http://iccss13.vfast.org/).

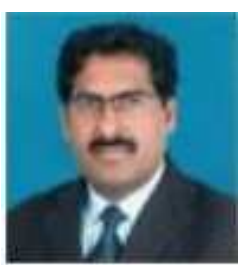

Nazir Ahmad Zafar $(\mathrm{PhD})$, was born in 1969 in Pakistan. He received his M.Sc. (Math. in 1991), M. Phil (Math. in 1993), and M.Sc. (Nucl. Engg. in 1994) from Quaid-i-Azam University, Pakistan. He received his $\mathrm{PhD}$ degree in computer science from Kyushu University, Japan in 2004. Currently, he is working as Associate Professor at College of Computer Sciences and Information Technology, King Faisal University, Saudi Arabia. His research interests are modelling of systems using mathematical approaches.

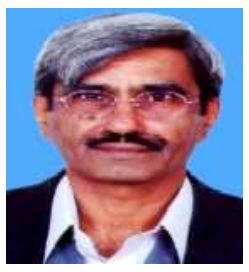

M. Akram (PhD), Is Chief Scientific Officer of COMSATS, Islamabad, Pakistan. His research interests are in the areas of applied mathematics, especially Newtonian, non-Newtonian fluid mechanics and bio mathematics. He has published research articles in reputed international journals of mathematical and engineering sciences. 